\title{
DAS MOBILISATIONSPOTENTIAL DER GEWERKSCHAFTEN IN MALAYSIA
}

Von RUdiger SielafF

Obwohl es Gewerkschaften als Institution in verschiedenartigen sozialen und politischen Systemen gibt, werden ihre Arbeitsweise und ihre Wirkungsmöglichkeiten nur selten miteinander verglichen. Weil abgesehen von einigen immer wiederkehrenden Grundfunktionen wenig Ubereinstimmung darüber herrscht, was Gewerkschaften eigentlich sind, gibt es wohl - zumindest meines Wissens - auch keine brauchbare Gewerkschaftstypologie.

Wann immer aber über Gewerkschaften geschrieben oder gesprochen wird, kann beim Autor/Redner ein der jeweiligen persönlichen Erfahrung angepaßtes Gewerkschaftsbild festgestellt werden, das im allgemeinen davon geprägt ist, Gewerkschaften als eine Vertretung von Arbeitern/Unterprivilegierten zu begreifen, die um die Besserung der wirtschaftlichen Lage und des politischen Einflusses der vertretenen Schichten bemüht sind.

Diejenigen also, die von den Gewerkschaften organisiert und vertreten werden, sind ebenfalls soziologisch im allgemeinen nur schwer zu umschreiben. Die Marxsche Gesellschaftsanalyse wird - ganz sicher zu Recht - nicht nur bezüglich ihrer Anwendbarkeit auf das Mitteleuropa des ausgehenden 20. Jahrhunderts in Frage gestellt, sondern muß in ihrem Wert sicher auch im Hinblick auf jene Länder bezweifelt werden, die man heute gemeinhin als Entwicklungsländer bezeichnet.

Dieser Aufsatz unternimmt den Versuch, das Phänomen ,,Gewerkschaft in einem Entwicklungsland" unter bestimmten Aspekten am Beispiel eines Landes zu beschreiben und einige Hinweise darauf zu geben, welche Rolle Gewerkschaften, die als Institution ja in den Jahren der europäischen Industrialisierung entstanden sind, im sozio-ökonomischen und politischen Prozeß der Entwicklungsländer spielen und/oder spielen können. Dabei wird bewußt auf einen theoretischen Rahmen verzichtet, weil 1. die empirischen Erkenntnisse des Verfassers zu begrenzt sind und 2. ein halbwegs umfassender theoretischer Rahmen anhand empirischer Daten nicht überprüft werden könnte. Denn entweder fehlen umfassende Daten ganz allgemein oder aber sie bauen in der Regel auf Erkenntnissen und Begriffen auf, die an Phänomenen der Industrieländer gewonnen und entwickelt wurden und dementsprechend in Entwicklungsländern gar nicht erhoben werden können.

Um das sozio-politische Potential der malaysischen Gewerkschaften, vielleicht auch mit einiger Vergleichbarkeit für andere Entwicklungsländer bestimmen zu können, soll hier nach einer Darstellung des Ambiente und der Grunddaten der malaysischen Gewerkschaften deren soziologische Struktur, ihre sozio-ökonomischen Errungenschaften sowie die politischen Ergebnisse ihrer Aktivitäten näher betrachtet werden. Abschließend soll dann eine Beschreibung versucht werden, in welchen Grenzen möglicherweise Gewerkschaften in Entwicklungsländern mobilisierend wirken können und welchen besonderen Bedingungen sie dabei unterliegen.

\section{Grunddaten der malaysischen Gewerkschaftsbewegung}

Die gegenwärtigen malaysischen Gewerkschaften wurzeln im Unterschied zu anderen asiatischen Gewerkschaften nicht in während der Kolonialzeit entstandenen und gegen die Kolonialmacht durchgesetzten Organisationen, wie z. B. in Indien oder Indonesien. Während der britischen Kolonialzeit haben sich in Malaya - und auch auf Borneo - kaum Gewerk- 
schaften gebildet, weil ihnen das Potential fehlte. Arme chinesische Einwanderer waren als Arbeiter nicht nur stark von traditionellen Konzepten bestimmt, sondern auch jederzeit von der Ausweisung bedroht. Die indischen Plantagenarbeiter unterlagen einer besonderen Gesetzgebung und waren deshalb auch nicht in der Lage, Gewerkschaften zu bilden. Die größte im Lande lebende Bevölkerungsgruppe, die Malaien, hatte, von wenigen Ausnahmen abgesehen, während der Kolonialzeit kaum Zugang zu modernen Beschäftigungen gefunden ${ }^{1}$. Erst im Laufe der dreißiger Jahre entstanden dann die ersten - wenn man so will - autochthonen Gewerkschaften. Diese wurden zumeist von Chinesen gebildet, die ihrerseits stark von den politischen Vorgängen in China ermutigt worden waren². Diese Gewerkschaften wurden bereits vor dem 2 . Weltkrieg von den englischen Kolonialherren als kommunistisch infiltriert bezeichnet und nach deren Wiederentstehung nach dem 2. Weltkrieg durch Reorganisationsmaßnahmen und Verbote rasch dezimiert ${ }^{3}$. Zu diesem Schritt sah sich die britische Kolonialmacht deshalb genötigt, weil die Malaiische Kommunistische Partei sich um die Erfolge der von ihr geführten Anti-Japanischen Volksarmee gebracht sah und unter Einbeziehung der von ihr kontrollierten Gewerkschaften einen Befreiungskampf gegen die Engländer begann. Die britische Kolonialmacht sah die einzige Möglichkeit, die kommunistische Machtübernahme zu verhindern, darin, das Vertrauen der im 2. Weltkrieg ebenfalls politisierten malaiischen und indischen Bevölkerungsgruppen zu gewinnen und die chinesische Bevölkerungsgruppe von der Malaiischen Kommunistischen Partei zu trennen und dagegen $\mathrm{zu}$ immunisieren.

Teil dieser britischen Bemühungen war es, in ihrer Grundtendenz anti-kommunistische Gewerkschaften zu fördern. Zu diesem Zwecke wurde in der Kolonialverwaltung neben dem Arbeitsministerium ${ }^{4}$ das besondere Amt eines „Trade Union Advisor of Malaya“ (TUAM) eingerichtet. Dieses sollte die Neuentstehung von Gewerkschaften ermutigen und deren politische Zuverlässigkeit sichern, wenn auch weniger durch administrative Maßnahmen als vielmehr durch Beratung und Hilfe ${ }^{5}$. Während also in anderen asiatischen Ländern das europäische Gewerkschaftskonzept schon frühzeitig den entsprechenden nationalen Bedingungen angepaßt wurde - womit keinerlei Urteil über diesen Vorgang verbunden werden soll, zumal in einigen Ländern diese Entwicklungen zu einer starken Zersplitterung der Gewerkschaften führten -, wurde in Malaysia das britische Modell eingeführt. Dabei ergab sich als Besonderheit, daß in einer multi-rassischen Gesellschaft die Malaien aus sozialstrukturellen Gründen und die Chinesen aus politischen Gründen nur ein geringes Gewicht in diese Gewerkschaften einbrachten. Als führende rassische Gruppe in den Gewerkschaften erstarkten deshalb die Inder.

Die so entstandenen Gewerkschaften bieten in ihrer Struktur ein buntes Bild und müssen schon deshalb in ihrer Wirkung und in ihrem Potential mit Vorsicht behandelt werden. Es gibt einige wenige, darunter vereinzelte große Industriegewerkschaften, eine große Zahl von Betriebsgewerkschaften und eine überaus große Anzahl, zumeist auch noch stark untergliederter Berufsgewerkschaften. Insbesondere diese strukturelle Zersplitterung erschwert den malaysischen Gewerkschaften die politische Koordinierung, auch wenn mit dem 1949 begründeten Malaysian Trades Union Congress (MTUC) nur eine nationale Gewerkschaftsdachvereinigung existiert.

1 Vgl. S. J. H. Zaidi: Malaysian Trades Union Congress 1949-1974, MTUC Petaling Jaya 1975, S. 1-6.

2 Vgl. Alex Josey: Trade Unionism in Malaya, Singapore 1958, 2nd rev. edition, S. 32 ff.

3 Vgl. The Trade Union Situation in the Federation of Malaya. Report of the Mission of the I. L. O., Genf 1962, S. $30-33$.

4 Während der Existenz des TUAM war das Arbeitsministerium eher ein Arbeitgeberministerium. Es verwundert wenig, daß diese Tradition beibehalten wurde, auch nachdem die TUAM-Kompetenzen dem Arbeitsministerium übertragen wurden.

5 Vgl. Charles Gamba: The Origins of Trade Unionism in Malaya, Singapore 1962. 
Grundsätzliche Bemühungen um eine einheitliche politische Ausrichtung werden immer wieder dadurch erschwert, daß verschiedene Organisationsprinzipien verschiedene Formen der Interessenvertretung und der Konfliktstrategie produzieren und diese dann wieder schlechter koordinierbar werden. Hinzu kommt, daß die Gewerkschaften zwar über ein multi-rassisches Grundkonzept verfügen, die politischen Parteien und die politische Kultur in ihrer rassischen Ausrichtung jedoch immer wieder störend einwirken.

Bei realistischer Einschätzung der verschiedenen vorhandenen Statistiken kann man gegenwärtig davon ausgehen, daß 1980 etwa 500000 Lohnempfänger in knapp 400 Gewerkschaften organisiert sind, davon allein 300000 in den 40 größten Gewerkschaften. Fast alle Gewerkschaften verzeichnen eine verhältnismäßig geringe Mitgliederdisziplin, d. h. die Zahl der Mitglieder schwankt ständig um ca. $50 \%^{6}$.

Die Organisationsrate liegt damit bezogen auf die erwerbsfähige Bevölkerung bei ungefähr $10 \%$, muß jedoch erheblich höher, wahrscheinlich bei ca. $20 \%$, veranschlagt werden, wenn man die Zahl derjenigen Erwerbstätigen ausschließt, die nach dem Gesetz nicht in Gewerkschaften organisiert werden dürfen? ${ }^{7}$.

Ein historischer Zahlenspiegel weist aus, daß die Organisationsrate der malaysischen Gewerkschaften seit Anfang der 50er Jahre eigentlich nicht mehr wesentlich gestiegen ist. Allerdings ist es den malaysischen Gewerkschaften immer wieder gelungen, ein durch krisenhafte Ereignisse ausgelöstes Absinken der Mitgliederzahlen wieder auszugleichen und im Saldo in den vergangenen 30 Jahren ein geringfügiges Wachstum zu erzielen.

\section{Die soziologische Struktur der malaysischen Gewerkschaften}

Der Versuch, den Standort der malaysischen Gewerkschaften in der Gesellschaft soziologisch zu bestimmen, muß in jedem Falle bruchstückhaft bleiben, weil intensive Untersuchungen zu diesem Thema nicht existieren und vorhandene Zahlen nur sehr grob gerastert sind. Allerdings ist es möglich, in zweierlei Hinsicht einige Aufschlüsse zu gewinnen: einmal können jene Bereiche, die von Gewerkschaften organisiert werden, etwas näher umschrieben werden, und andererseits sind aufgrund der Kenntnisse über die einzelnen Gewerkschaften auch Aussagen darüber möglich, welche Arbeitnehmergruppen von den einzelnen Gewerkschaften repräsentiert werden.

Betrachtet man den Organisationsgrad in den verschiedenen Bereichen der malaysischen Wirtschaft, so ergibt sich folgendes Bild:

\footnotetext{
6 So äußern sich viele Gewerkschaftsführer. Vor Abschluß eines in der Regel 3jährigen Tarifvertrages steigt die Mitgliederzahl gewöhnlich stark an, um dann rasch zu fallen.

7 Vgl. die einleitenden Paragraphen des ,,Industrial Relations Act 1967“.
} 
Gewerkschaftlicher Organisationsgrad in verschiedenen Wirtschaftssektoren Malaysias, 1971 und 1974 (in \%) ${ }^{8}$

Landwirtschaft, Forsten, Fischerei

$1971 \quad 1974$

Landwirtschaftliche Produktion

$1 \% \quad 1 \%$

mit Bearbeitung (Plantagen)

$11 \% \quad 17 \%$

Bergbau

$13 \% \quad 18 \%$

Verarbeitende Industrie

$12 \% \quad 21 \%$

Bauindustrie

$1 \% \quad 2 \%$

Elektrizitäts- und Wasserversorgung

$43 \% \quad 52 \%$

Handel, Banken

$6 \% \quad 8 \%$

Transport, Nachrichtenwesen

$36 \% \quad 31 \%$

Dienstleistungen

$25 \% \quad 31 \%$

Insgesamt

$12 \% \quad 14 \%$

Die Entwicklung von 1971 bis 1974 ist verhältnismäßig günstig für die Gewerkschaften, weil nach der Notstandsphase 1969-1971 infolge der Auflösung einiger Gewerkschaften und des Drucks auf die Gewerkschaften allgemein generell die Zahl der Gewerkschaftsmitglieder zurückging und sich in der Folgezeit langsam wieder erholte. Nach 1974 dürfte die Entwicklung weiter, wenn auch langsamer vorangegangen sein.

Trotzdem ist auffällig, daß im Bereich ,,Landwirtschaft, Forsten, Fischerei“ so gut wie keine Gewerkschaften existieren. Dieses ist darauf zurückzuführen, daß die aktive Bevölkerung dieses Wirtschaftssektors als selbständig gilt und nach dem Gesetz nicht in Gewerkschaften organisiert werden darf. Der verhältnismäßig niedrige Organisationsgrad bei „,Handel und Banken“ sowie „Bauindustrie“ ist auf die große Anzahl von Kleinbetrieben und auf traditionelle Beschäftigungsmethoden zurückzuführen, wodurch die Gewerkschaftsbildung erschwert wird.

Beachtlich ist der Anstieg der Mitgliedschaft im Bereich der verarbeitenden Industrie, der eben nicht nur absolut, sondern in seiner relativen Größe darauf zurückführbar ist, daß hier eine auch nach europäischen Normen klassische Schicht von Fach- und angelernten Arbeitern entsteht.

Der relativ niedrig erscheinende Organisationsgrad für die Bereiche „Plantagen“ und „,Bergbau“ darf nicht falsch eingeschätzt werden. In beiden Bereichen herrschen noch Beschäftigungs- und auch Siedlungsformen vor, die aus der kolonialen Tradition kommen und die Organisation von Gewerkschaften sehr erschweren. Außerdem schwankt in diesen Bereichen die Mitgliedschaft stark.

Der hohe Organisationsgrad in den Bereichen ,,Dienstleistungen“, ,,Transport, Nachrichtenwesen“ und „Elektrizitäts- und Wasserversorgung" ist mit davon beeinflußt, daß diese Bereiche in starkem Maße zum öffentlichen Dienst zählen und daß in diesen Bereichen relativ hohe berufliche Eingangsqualifikationen erforderlich sind, die wiederum die Bildung von Gewerkschaften erleichtern. Denn, wie auch weiter unten ausgeführt wird, stehen Bildungsgrad und Organisationsgrad offenbar in engem Zusammenhang.

Es ist festzustellen, daß die Gewerkschaften bis auf die Land- und Forstwirtschaft sowie das Fischereiwesen sämtliche Bereiche der Volkswirtschaft ausreichend erfassen. Auch ist diese

8 Tab. 6 in: Rüdiger Sielaff: Malaysia - Gewerkschaften, Wandlungen im Selbstverständnis einer gesellschaftlichen Gruppe, Arbeiten aus der Abteilung Entwicklungsländerforschung des Forschungsinstituts der Friedrich-Ebert-Stiftung, Nr. 37, Juli 1976, S. 203. 
,gesellschaftliche Lücke“ durch die Gesetzgebung hervorgerufen. Legt man die gesamte arbeitsfähige Bevölkerung zugrunde, so ist ein Sektor von ca. $25 \%$ dieser Bevölkerung nicht durch Gewerkschaften repräsentiert. Gemessen an anderen, in ihrer Grundstruktur landwirtschaftlichen Ländern Asiens, ist daher der Grad der gewerkschaftlichen Organisation in Malaysia als hoch anzusehen. Hinsichtlich der Repräsentation ist festzuhalten, daß die malaysischen Gewerkschaften zwar eine gewichtige Gruppe der Gesellschaft nicht vertreten, daß sie aber andererseits auch nicht eine Vertretung von Minderheiten sind.

Betrachtet man den beruflichen Status der arbeitsfähigen Bevölkerung in Malaysia, so ergibt sich - auch wenn der gegenwärtige Trend noch stärker zu den abhängig Beschäftigten gehen dürfte, wofür hier keine exakten Zahlen vorgelegt werden können - anläßlich der letzten Volkszählung im Jahre 1970 folgendes Bild:9

Arbeitgeber

Selbständige

Arbeitnehmer

Unbezahlte Familienangehörige etc.
$4 \%$

$29 \%$

$50 \%$

$17 \%$

Auch wenn dies nur mit Hilfe von Zahleninterpretationen möglich ist, soll hier versucht werden, die genannte Struktur der arbeitsfähigen Bevölkerung mit der Struktur der Gewerkschaften zu vergleichen. Die Gewerkschaften haben keine Zahlen über ihre Mitgliederstruktur bzw. machen diese nicht zugänglich, so daß wir darauf angewiesen sind, die vorhandenen Gewerkschaften entsprechend den von ihnen organisierten Mitgliedern zu kategorisieren. Dabei ergibt sich folgendes Bild: ${ }^{10}$

Gewerkschaften, die den gehobenen Verwaltungsdienst,

Lehrer, Techniker und Akademiker organisieren

62818 Mitgl.

Gewerkschaften, die Verkaufspersonal, Büropersonal und einfache technische Angestellte organisieren

Gewerkschaften, die Hand- und Fabrikarbeiter organisieren

47831 Mitgl.

Kombinierte Gewerkschaften, die alle Kategorien organisieren

175950 Mitgl.

7764 Mitgl.

Gewerkschaften, die Lehrer, Techniker, Akademiker

und Büropersonal organisieren

1208 Mitgl.

Gewerkschaften, die Büropersonal und Handarbeiter organisieren

89506 Mitgl.

Betriebsgewerkschaften, die vorwiegend

Büropersonal und Fabrikarbeiter organisieren

28430 Mitgl.

Berücksichtigt man nur die ersten drei, weil eindeutigen Kategorien, bleibt zwar ca. ein Drittel der Gewerkschaftsmitgliedschaft unberücksichtigt, doch ergibt sich ein sicherlich repräsentatives Bild über die Mitgliederstruktur der Gewerkschaften:

Hand- und Fabrikarbeiter 
Die gehobenen Berufsgruppen heben sich allgemein aus der Gewerkschaftsszene ab und gehören mit ihren Verbänden zumeist nicht dem MTUC an.

Im Vergleich dazu ergibt sich aufgrund der Berufsstruktur der beschäftigten Bevölkerung folgende Verteilung: ${ }^{11}$

Hand- und Fabrikarbeiter

$80 \%$

Verkaufs- und Büropersonal

$14 \%$

Lehrer, Techniker, Akademiker

Dieser knappe Zahlenvergleich zeigt bereits deutlich, daß tendenziell die Hand- und Fabrikarbeiter schlechter organisiert sind als das Büro- und Verkaufspersonal, das in beiden Verteilungsstatistiken etwa gleich stark vertreten ist. Hingegen ist das qualifizierte Personal überdurchschnittlich hoch organisiert, da es mit $6 \%$ Beschäftigungsanteil $22 \%$ der Organisierten stellt.

Scheint diese Gruppe in der Mitgliedschaft der Gewerkschaften bereits einflußreich, so verstärkt sich dieses Bild noch, wenn man die gewerkschaftlichen Führungsgremien betrachtet. Zwar liegen keine umfassenden empirischen Daten für einzelne Gewerkschaften vor, doch ist es sicherlich nicht verfehlt, die Zusammensetzung der Führungsgremien des Malaysischen Gewerkschaftsbundes (MTUC) insofern für repräsentativ für die Führungsstruktur der Gewerkschaften zu halten, als er im Vorstand die Spitzenvertreter der Einzelgewerkschaften versammelt und überdies dort die größeren Gewerkschaften über eine stärkere Repräsentanz verfügen.

Für die drei Vorstände zwischen 1971 und 1976 (die Gesamtzahl der Miglieder des erweiterten Vorstands stieg) ergibt sich folgendes Bild: ${ }^{12}$

\begin{tabular}{lrrr}
\hline & $1971-72$ & $1973-74$ & $1975-76$ \\
\hline Berufsgewerkschafter/-politiker & $25 \%$ & $23 \%$ & $19 \%$ \\
Handarbeiter/Fabrikarbeiter & $18 \%$ & $19 \%$ & $19 \%$ \\
Büro- und Verkaufspersonal & $29 \%$ & $32 \%$ & $39 \%$ \\
Lehrer, Techniker, Akademiker & $26 \%$ & $26 \%$ & $24 \%$ \\
Andere, ohne Angaben & $2 \%$ & - & - \\
\hline
\end{tabular}

Diese Zahlenangaben bestätigen die Beobachtung, die man in den Gewerkschaften selbst machen kann, daß nämlich die Arbeitnehmer mit qualifizierterer Ausbildung die Gewerkschaften beeinflussen und ihre Führung stellen, daß aber die Hand- und Fabrikarbeiterschaft gleichbleibend wenig repräsentiert ist. Diese Tendenz ist im übrigen auch gerade in Betriebsgewerkschaften zu beobachten.

11 Die Zahlen wurden ermittelt nach: Statistical Handbook of Peninsular Malaysia 1974, Kuala Lumpur 1975, S. 7, und dann mit den eindeutig zuweisbaren Zahlen auf S. 7 verglichen.

12 Aufstellung nach eigenen Unterlagen. 


\section{Soziale und wirtschaftliche Ergebnisse der Gewerkschaftsaktivität}

Die soziologische Struktur der Gewerkschaftsmitgliedschaft wie auch der Gewerkschaftsführungen, wie sie hier anhand einiger Zahlen grob skizziert werden konnte, kann sicherlich nicht als Beweis dafür dienen, daß die malaysischen Gewerkschaften von der Mittelklasse oder mittelklasse-ähnlichen Gruppen beherrscht würden oder gar deren Interessen dienten. Denn auch unter starker Beteiligung von Vertretern der Mittelklasse können Gewerkschaften eine Vertretung der breiten abhängigen Arbeitnehmerschaft sein bzw. sich zu einer solchen entwickeln (zumal in der Kolonialzeit, die ja noch nicht allzu lange vergangen ist, es Mittelklasse-Elemente waren, die sich als erste deutlich gegen die Kolonialmacht organisierten). Um die Rolle der genannten einflußreichen Gruppen innerhalb der Gewerkschaften eindeutiger beschreiben zu können, sollen daher einige gewerkschaftliche Aktivitäten näher betrachtet werden.

In diesem Zusammenhang ist zunächst zu erwähnen, daß es Ansätze zu einer solchen Diskussion innerhalb der malaysischen Gewerkschaften gegeben hat und noch gibt. Im Laufe der Jahre 1976 und 1977 fand innerhalb des Verbandes der Gewerkschaften des öffentlichen Dienstes (CUEPACS) eine längere Debatte statt, in der die Hand- und Bauarbeiter des öffentlichen Dienstes gemeinsam mit dem Büropersonal gegen die Verhandlungsführung ihres Verbandes mit der Begründung protestierten, die dort sitzenden Lehrer und Techniker sorgten für ihre eigenen Interessen und vernachlässigten die Interessen der weniger qualifizierten - und auch schlechter bezahlten - Kräfte.

Ähnliche Überlegungen veranlaßten 1976 die Gewerkschaften aus dem Bereich der privaten Wirtschaft zur Bildung eines neuen Verbandes, weil sie den öffentlichen Dienst für besser organisiert hielten und die Interessen der industriellen Arbeitnehmerschaft in der Offentlichkeit wie auch innerhalb des MTUC nur ungenügend vertreten sahen ${ }^{13}$.

Als Hauptaktivitäten betrachten die malaysischen Gewerkschaften - entsprechend ihren klassischen Vorbildern in Europa und Nordamerika - die Verbesserung der Lohn-, Lebensund Arbeitsbedingungen der Arbeitnehmer. Aufgrund des Arbeitsmarktes von Malaysia, auf dem die qualifizierten Kräfte knapp sind, bedarf es wohl keiner langen Ausführungen, daß die genannten ,,mittelklasseverdächtigen“ Arbeitnehmergruppen wenig Mühe hatten, ihre Einkommensverhältnisse so zu gestalten, daß sie keine Einkommensverluste oder ein Absinken ihres Lebensstandards hinzunehmen hatten.

Im Hinblick auf eine breite Mobilisierung der Arbeitnehmerschaft ist es wichtiger zu überprüfen, ob die Gewerkschaften in der Lage waren und sind, die Lebensbedingungen für die unteren Einkommensgruppen innerhalb der Arbeitnehmerschaft zu verbessern. Als wichtigstes Instrument in der Realisierung dieses Ziels können auch in Malaysia Tarifverträge gelten, die vor allem im Bereich der produzierenden Industrie und der Plantagen zur anerkannten Praxis geworden sind, Lohn- und Arbeitsbedingungen festzusetzen.

Betrachtet man kursorisch die Situation der Plantagenarbeiter in Malaysia, so wird die heutige Generation der Plantagenarbeiter durch eine mitgliederstarke Gewerkschaft vertreten, und ihre Lohn- und Arbeitsbedingungen werden weitgehend durch die Tarifverträge dieser Gewerkschaft bestimmt. Noch die Väter und Großväter dieser Plantagenarbeitergeneration waren aufgrund einer kolonialen Gesetzgebung ins Land gekommen, die auch die allgemeinen Bedingungen festschrieb, ihnen kaum soziale Vergünstigungen ließ und sie in ihren Lohn-, Wohn- und Lebensbedingungen fast völlig von ihrem Plantagen-Arbeitgeber abhängig machte. Die laufenden Tarifverträge verbesserten das Los der jüngsten Generation

13 Eigene Beobachtung, vgl. auch die laufende Zeitungsberichterstattung. 
schrittweise: Während es noch 1954 keine regelmäßigen wöchentlichen Ruhetage für Plantagenarbeiter gab, verfügen sie seit 1964 über einen freien Tag bei vollem Lohnausgleich; während 1954 zwei Wochen Jahresurlaub zumeist als zusätzliche Vergütung ausgezahlt wurden, beträgt dieser seit 1964 drei Wochen und seit 1972 sogar 31/2 Wochen; während ein Plantagenarbeiter 1954 bei halber Bezahlung maximal zwei Wochen im Jahr erkranken durfte, kann er seit 1964 bis zu 90 Tagen bei halber Bezahlung und seit 1972 gar bei voller Bezahlung erkranken, ohne automatisch seinen Arbeitsplatz zu verlieren ${ }^{\mathbf{1 4}}$.

Auch die Löhne entwickelten sich in der Zwischenzeit:

Tagelohn von Plantagenarbeitern ${ }^{15}$ 1954-1974

(Jahresinflation durchschn. $2 \%$ )

\begin{tabular}{lccc}
\hline & 1954 & 1964 & 1974 \\
Tagelohn eines Kautschukzapfers & $\mathrm{M} \$ 2,40$ & $\mathrm{M} \$ 3,55$ & $\mathrm{M} \$ 6,73$ \\
Feldarbeiter & $\mathrm{M} \$ 2,05$ & $\mathrm{M} \$ 3,10$ & $\mathrm{M} \$ 4,84$
\end{tabular}

Auch im industriellen Bereich haben Gewerkschaften ähnliche Erfolge für den ungelernten und den angelernten Arbeitnehmer erzielt. Die Durchschnittslöhne für verschiedene Industrien, in denen die Arbeitnehmer organisiert und durch Tarifverträge geschützt sind, und für nichtorganisierte Industrien mögen dies nachweisen:16

Monatlicher Durchschnittslohn gelernter und ungelernter Arbeiter in ausgewähleten Industrien 1971 (in M\$)

Durchschnittlicher Monatslohn

$\begin{array}{cc}\text { Gelernte } & \text { Ungelernte } \\ \mathrm{M} \$ 159,29 & \mathrm{M} \$ 100,34\end{array}$

a) Gewerkschaftlich organisierte Industrien

Kautschukindustrie

$M \$ 121,21 \quad M \$ 107,19$

Nahrungsmittelindustrie

M\$157,27

$\mathrm{M} \$ 103,56$

Getränkeindustrie

$\mathrm{M} \$ 145,76$

$\mathrm{M} \$ 109,54$

Tabakindustrie

$\mathrm{M} \$ 157,23$

$\mathrm{M} \$ 134,81$

Holzindustrie

M\$ 140,65

M\$ 86,70

Chemieindustrie

M\$192,85

$\mathrm{M} \$ 137,28$

Metallindustrie

$\mathrm{M} \$ 250,21$

$\mathrm{M} \$ 170,33$

b) Gewerkschaftlich nicht/kaum organisierte Industrien

Lederindustrie

$\mathrm{M} \$ 118,43 \quad \mathrm{M} \$ 76,96$

Textilindustrie

M\$ 91,69 M\$ 64,80

Schuhindustrie

M\$ 87,37

M\$ 55,45

Bauindustrie

$\mathrm{M} \$ 348,15$

$\mathrm{M} \$ 118,30$

Versch., einschl. elektronische Industrie

$\mathrm{M} \$ 104,76$

$\mathrm{M} \$ 79,56$

Diese Tabelle zeigt deutlich an, daß in gewerkschaftlich organisierten Industrien das Lohnniveau höher ist als in nichtorganisierten Industrien, wenngleich der Fall der Bauindustrie ausweist, daß auch der ,,Markt" die Löhne nach oben reguliert. Angemerkt sei auch noch,

14 Malaysian Trades Union Congress, General Council Report 1965-1966, Kuala Lumpur, S. 21-22; Introducing the National Union of Plantation Workers, Report of the Research Department, NUPW, Broschüre o. O., o. J. (1975); National Union of Plantation Workers, General Report 1969-1971, Kuala Krai, S. 71 ff.

15 Malaysian Trades Union Congress, General CouncilReport 1965-1966, Kuala Lumpur, S. 21-22; I. L. O. StatisticalYearbook 1975. 16 Wage Structure in Different Industries in Malaysia, Research Department MTUC, December 1974. 
daß in den meisten malaysischen Industrien ,, contract workers “ beschäftigt sind, die in der Regel durch Mittelsmänner auf Tagesbasis ausgeliehen werden, keinerlei Sozialgesetzgebung unterliegen, gesetzlich als selbständig gelten und daher nicht organisiert werden können und zumeist noch zwischen 20 und $50 \%$ weniger verdienen als die ungelernten Arbeitskräfte.

Andererseits weist diese Statistik aus, wie breit die Schwankungen um den Durchschnitt sind, d. h. wie stark auch durch gewerkschaftliche Aktivitäten eine Lohnhierarchie geschaffen wird.

Auf der Basis der hier zitierten Statistiken kann man sicher davon ausgehen, daß die malaysischen Gewerkschaften die materiellen und sozialen Interessen der Arbeitnehmer - wenn auch nicht aller - erfolgreich vertreten.

Wie stark jedoch gewerkschaftliches Denken, d. h. das Bewußtsein, durch gewerkschaftliche Aktion etwas zu erreichen, in Malaysia wirkt, sollte weniger an diesen Zahlen abgelesen werden als vielmehr an einer Schlußfolgerung, zu der N. Danaraj in einer soziologischen Untersuchung über einen Streik anläßlich der Nichtanerkennung einer Gewerkschaft gekommen ist. Dieses Zitat zeigt, wie sehr unter Arbeitnehmern dieses Bewußtsein spontan vorhanden war - aber offenbar doch aus der Erfahrung des Beispiels anderer. Danaraj schreibt ${ }^{17}$ : "It was the solidarity of the workers that gave spirit to the leaders. What was unique in the growth of this movement was that it sprung not by any premeditated attempt by any group of ardent unionists but it was the product of disillusionment in the hearts of workers. Non of the workers had ever been involved in union activities nor did they conceive their unity as an attempt to challenge the power and position of the management. They only wanted some of their conditions in the factory to be changed."

Auch wenn man im Hinblick a uf die soziologische Struktur der Gewerkschaften, insbesondere der Gewerkschaftsführungen, zu dem Schluß gelangt, daß Mittelklasse-Gruppen eine starke Rolle im Bereich der Gewerkschaften spielen, diese auch sicher in der Vergangenheit ihre Interessen gut vertreten haben, so ist auch zu schlußfolgern, daß das Grundkonzept von Gewerkschaften weit verbreitet ist und sich materiell und sozial für die ungelernten und gelernten unteren Arbeitnehmergruppen positiv ausgewirkt hat.

\section{Die Gewerkschaften und nicht-organisierte Unterprivilegierte}

Die ,ländlichen Armen“ haben die Phantasie der asiatischen Gewerkschafter seit einer Reihe von Jahren beschäftigt, so auch in Malaysia. So hat z. B. P. P. Narayanan, Generalsekretär der Plantagenarbeitergewerkschaft (NUPW), heute Präsident des MTUC und des Internationalen Bundes Freier Gewerkschaften (IBFG), bereits am 21.2. 1966 in einer Rede eingestanden, daß in den meisten Ländern Asiens und Afrikas Bauern, Landarbeiter und Fischer nicht in Gewerkschaften organisiert werden könnten, daß die Gewerkschaften unglaubwürdig werden würden, wenn sie nichts für diese Gruppe täten. Er hielt es deshalb für notwendig, diese Gruppen in Genossenschaften zu organisieren und mit Hilfe einer $\mathrm{Zu}$ sammenarbeit dieser Genossenschaften mit den Gewerkschaften eine neue Massenbewegung zu schaffen ${ }^{18}$. Diesbezüglich wurden jedoch in Malaysia keinerlei Fortschritte erzielt. Diesem stand vor allem der politische Selbstbehauptungswille der Regierungspartei entgegen. Die führende Regierungspartei, die United Malay National Organization (UMNO),

17 N. Danaraj: The Law on the Right to Strike and the Case of Southeast Asia Firebricks, University of Malaya, 1976, S. 41. 18 Zit. n. J. Victor Morais: Narayanan - The Asian Trade Union Leader, Petaling Jaya 1975, S. 141. 
hat ihre politische Basis auf dem Lande und wacht eifersüchtig darüber, daß diese Machtbasis erhalten bleibt und nicht unter fremden Einfluß gerät ${ }^{19}$.

Andererseits hat die erfolgreiche Politik der malaysischen Regierung, neue landwirtschaftliche Anbauflächen zu erschließen - und bevorzugt an die malaiische Bevölkerung zu verteilen -, für ein neues gewerkschaftliches Problem auf dem Lande gesorgt. In zunehmendem Maße sind die Besitzer größerer Flächen unter diesen Neuansiedlern dazu übergegangen, zumindest für die Erntezeit Landarbeiter zu beschäftigen. Da in den jeweiligen Betrieben maximal zwei bis drei Arbeiter beschäftigt werden, die Gewerkschaften es jedoch mit einer großen Zahl von Einzelbauern zu tun haben, sind bisher alle Versuche, diese Landarbeiter in gewerkschaftlichen Organisationen zu erfassen, vergeblich gewesen ${ }^{20}$.

Um nun auf anderem Wege sich an der Lösung dieser Probleme zu beteiligen und andererseits Beiträge zur allgemeinen Entwicklung des Landes, besonders einiger sozialer Randerscheinungen zu leisten, haben die malaysischen Gewerkschaften eine Politik entwickelt, die ökonomische Aktivität der Gewerkschaften zu steigern ${ }^{21}$ und ihre Kapazitäten zur Ausbildung der Kinder von Gewerkschaftsmitgliedern und ländlichen Armen zu erweitern ${ }^{22}$. So wurden gewerkschaftliche Stipendienfonds und auch gewerkschaftseigene Ausbildungsstätten geschaffen, vor allem aber eine Reihe gewerkschaftseigener Wirtschaftsunternehmen und Genossenschaften.

Auch wenn diese Politik gewerkschaftsintern umstritten ist (vor allem, weil den älteren Gewerkschaftsführern unterstellt wird, sie wollten sich Denkmäler setzen und/oder bereichern) und ein Urteil über die Wirksamkeit dieser Aktivitäten verfrüht wäre, so darf dieser neu entwickelten Politik weder die Absicht noch die erfolgreiche Tendenz abgesprochen werden, daß sie die Basis der Gewerkschaften erheblich verbreitert und ihren Einfluß im Lande konsolidieren wird.

\section{Die malaysischen Gewerkschaften und die Politik}

Obwohl eine Reihe von Gewerkschaftsvertretern nach 1950 bis zu den ersten Wahlen 1959 in den Gesetzgebenden Rat der Malaiischen Föderation berufen worden waren, haben die Gewerkschaften als Institution ein direktes politisches Engagement unterlassen. Zwar hat es immer wieder politische Aktivitäten einzelner Gewerkschaftsführer gegeben ${ }^{23}$, doch haben die Gewerkschaften selbst den Kontakt zu den grundsätzlich rassisch orientierten politischen Parteien beinahe ängstlich gemieden.

Für die Grundhaltung der malaysischen Gewerkschaften typisch ist die folgende Äußerung des derzeitigen Präsidenten des MTUC und sicherlich einflußreichsten Gewerkschafters in Malaysia, P. P. Narayanan: ${ }^{24}$

"There are issues that can be solved by negotiations, conciliation or arbitration. There are also issues that have to be solved only by political action. But the democratic experiment in Southeast Asia is so new and so young that nobody can stop the tendency of the rulers to ban

\footnotetext{
19 Dies wirdvon Gewerkschaften als Grund für ihre diesbezügliche Inaktivität genannt. Als die Jugendsektion des MTUC vom 12. bis 16. Oktober 1976 eine Studienreise durch die ländlichen Gebiete der Ostküstenstaaten machte, bekam sie diese Politik konkret zu spüren, als ihr der Zugang zu Dörfern nicht gestattet wurde.

20 So der Hinweis von NUPW-Führern.

21 Vgl. P. P. Narayanan: Social and Economic Contributions of Rural Unions to Development, in: Rural Unions Contribution to Socio-Economic Development, edited by Ruediger Sielaff, Petaling Jaya 1976, S. 34.

22 Vgl. Bericht der ,National Union of Plantation Workers“, in: Rural Unions Contribution ..., a. a. O., S. 96-97.

23 Vgl. Rüdiger Sielaff, a. a. O., S. 35 ff. - Der gegenwärtige Generalsekretär des MTUC, V. David, sitzt für die Oppositionelle DAP im Parlament.

24 Zit. n. J. Victor Morais, a. a. O., S. 141.
} 
unions that are in sympathy with opposition political parties. Trade unions generally swing to the side of socialist or liberal parties. If such parties are not in existence they tend to form one ... I must hasten to say that even if labour reaches a stage when the labour movement is ready to support a party of its choice, the organic independence of trade unions must be maintained. Otherwise the trade union movement will be pulled into the vortex of the political machinery and workers' freedom will be completely lost."

Diese grundsätzliche Äußerung macht deutlich, daß für die malaysischen Gewerkschafter das Problem der politischen Rolle der Gewerkschaften unter drei Aspekten wichtig ist; denn auch wenn einige Aspekte immer wieder kontrovers diskutiert wurden, so haben die meisten Gewerkschaftsführer in der Praxis Narayanans Einschätzung geteilt.

Grundsätzlich wird also anerkannt, daß zur Erreichung gewisser gewerkschaftlicher Zielsetzungen es der politischen Aktion bedarf. Allerdings wird mit Rücksicht auf die Verhaltensweise der Regierung die Unterstützung bestimmter politischer Parteien ausgeschlossen, ja grundsätzlich für alle Zukunft die organische Unabhängigkeit von politischen Parteien gefordert, um die Einheit der Gewerkschaften nicht aufs Spiel zu setzen. Das indische Beispiel sowie die indonesischen Erfahrungen vor 1965 werden immer wieder zur Abschreckung zitiert. Aufgrund der parteipolitisch bunten Zusammensetzung der Gewerkschaften wäre eine einseitige Bindung an eine bestimmte Partei auch sicherlich das Ende der gegenwärtigen Gewerkschaften.

Das politische Feld, das die Gewerkschaften besonders unmittelbar betrifft, ist die Arbeitsgesetzgebung. Auf diesem Gebiete haben die malaysischen Gewerkschaften in den vergangenen 15 Jahren viel getan, auch wenn die verschiedenen Berichte des MTUC immer wieder ausweisen, daß die Mißerfolge zahlreicher waren als die Erfolge. Ganz sicher hat es in diesem Bereich auch eine Mobilisierung gegeben, zumal die Gewerkschaften in anderen politischen Fragen noch weniger zu mobilisieren waren.

Seit 1965 - seit in einer Notstandsphase zunächst als Essential Regulations und dann als Industrial Relations Act eine neue Gewerkschaftsgesetzgebung eingeführt wurde - haben die malaysischen Gewerkschaften nach Mitteln und Wegen gesucht, sich der Einschränkungen, denen ihre Arbeit im Laufe der Zeit unterworfen worden war, zu entledigen. Sie erwiesen sich jedoch als zu schwach, um der Regierung größereZugeständnisse abzuringen. Die Resignation, die sich da auf gewerkschaftlicher Seite ergeben hat, dokumentiert sich auch in den Berichten des MTUC-Generalsekretärs. Er schreibt 1966:25 "Malaysia has now become the employer's paradise. Spoon-fed under the pioneer status, protected under the Federal Laws and their interests specially looked after by the Ministry of Labour, it is no wonder that many of them have become arrogant, anti-union and have increasingly shown tendency to act as overlords of medieaval periods. The essential regulations have been outrageously exploited and that too with the seemingly active connivance of the government officials. Apparently this is what the government means by an open society with free flow of foreign capital."

Und ein Jahr später heißt es resignierend:26 "Thus, the essential regulations relating to trade unions which were said to be temporary in nature are likely to become the permanent feature of the government's industrial relations policy. Despite the promises, public declarations and sweet assurances during meetings, there is no sign that the regulations will ever be withdrawn. The government has finally shown its true colours which spell disaster for the workers. If even now they remain indifferent, disinterested and unwilling to accept their obliga-

25 Malaysian Trades Union Congress, General Council Report 1965-1966, Kuala Lumpur, S. 6.

26 Malaysian Trades Union Congress, General Council Report 1966-1967, Kuala Lumpur, S. 125. 
tions and responsibilities, than the possibilities of their salvation will become all the more remote."

Hier wird also unumwunden eingestanden, daß die Regierung in der Lage war, mit Hilfe gesetzlicher Regelungen und unter dem Bruch eigener Versprechungen den Spielraum der Gewerkschaften empfindlich einzuengen, ohne daß diese viel dagegen unternommen hätten. In der Notstandsphase 1969-1971 verschärften sich die Zustände sogar noch, d. h. die Möglichkeiten der Gewerkschaften, eine Änderung der abgelehnten Gesetzgebung durchzusetzen, wurden weiter verringert. Doch brach in der Erholungsphase der Gewerkschaften, die sich im erneuten Zuwachs der Mitgliederzahlen nach 1971 abzeichnet, eine Debatte auf, die einige der Gründe für diese Schwäche der Gewerkschaften offenlegt. Diese Debatte wurde sehr selbstkritisch geführt und führte den MTUC in eine Krise, in der heftig um den politischen Kurs gerungen wird und die auch nach der Delegiertenversammlung 1980 keineswegs beendet ist.

Am 4. Mai 1977 überreichte eine Gruppe von Gewerkschaften dem Generalsekretär des MTUC eine Denkschrift, in der es lapidar hieß:27 "An analysis of the developments during the past decade will inevitably lead one to the conclusion that workers and their organizations have lost more than what they have achieved. The labour laws have been continuously amended detrimental to the workers and their unions. In the public sector the unions have been systematically denied of collective bargaining. Vast majority of the workers remain unorganised. MTUC by shirking its responsibility have become responsible for this deterioration.

As we scrutinize the frustration of the affiliated unions, we come across several reasons. We list hereunder some of them:

The manner in which MTUC leadership handle important and serious problems faced by affiliated unions show quite clearly that the leaders at the helm of MTUC are not in the least bothered.

They merely take up the matter because of persistance of affiliated unions.

Loss of spirit of sacrifices and dedication and instead a love for materialism has crept in. There is a glaring tendency to tow the line of the government irrespective of consequences." Außer dieser Globalkritik gab e s nur vorsichtige Andeutungen, keinesfalls öffentliche Äußerungen, daß einige Gewerkschaftsführer sich zu Vollstreckungsgehilfen der Regierung gemacht hätten. Vor allem der größten malaysischen Gewerkschaft, der National Union of Plantation Workers (NUPW), wurde der Vorwurf gemacht, durch vorsichtiges Taktieren die Chance für eine massive Gegenaktion vertan zu haben. Tatsächlich hat nach dem Eingeständnis ihrer eigenen Führer die NUPW gegenüber der Regierung immer vorsichtig taktiert, weil sie am meisten zu verlieren hatte, weil sie ihre Organisation nicht gefährden wollte und weil sie sich im Rahmen der politischen Gepflogenheiten jener Jahre als erpreßbar erwies. Angesichts der Notstandsregulierungen von 1969-1971 war die NUPW vor allem darum bemüht, für Zehntausende von Plantagenarbeitern ohne malaysischen Paß die plötzlich vorgeschriebenen Arbeitserlaubnispapiere zu erwirken und deren Ausweisung zu verhindern.

Auch repräsentiert sie den größeren Teil der rassischen Gruppe der Inder, die politisch von der an der Regierung beteiligten Inder-Partei, dem Malaysian Indian Congress (MIC), repräsentiert wird. Der MIC stellte die längste der zur Debatte stehenden Zeit den Arbeitsmi-

27 Memorandum der: Union of Employees in Trade Unions, Metal Industry Employees' Union, Machinery Manufacturing Employees' Union, Kesatuan Pekerja2 Kumpulan United Motor Works, Non-Metallic Mineral Products Manufacturing Employees' Union, Kesatuan Pekerja2 Perusahaan Membuat Tekstil dan Pakaian Perak, Kesatuan Pekerja2 Perusahaan Membuat Tekstil dan Pakaian Selangor, National Union of Employees in Companies Manufacturing Rubber Products; Kopie im Besitz des Verfassers. 
nister. Ein Streit zwischen dem politischen und dem gewerkschaftlichen Führer der Inder hätte zwangsläufig zu einer Schwächung des indischen Einflusses führen müssen ${ }^{28}$. So waren der Gewerkschaftsführung konkrete Interessen einer großen Gruppe wichtiger als gewerkschaftliche Grundsatzfragen. Dieser Ausgleich aber zwischen divergierenden Interessen unter den Mitgliedern ist auch bei großer Argumentationsakrobatik nur schwer vollziehbar. Auch stillschweigende Abkommen sind unter solchen Voraussetzungen nur noch durch Doppelzüngigkeit zu halten. Tatsächlich haben auch Politiker darüber Beschwerde geführt, daß Gewerkschaftsführer in öffentlichen Äußerungen die Regierung hart kritisierten, diese aber hinterher am Telefon relativierten und mit der Bemerkung versehen, man müsse den Mitgliedern Zugeständnisse machen ${ }^{29}$.

Auch der frühere Generalsekretär des MTUC, S. J. H. Zaidi, machte auf dieses Problem aufmerksam. Er sagte in einer Rede am 17. 12. 197730:

"Not to speak up under these circumstances is a betrayal of trust which the working class has placed in its leaders. However, hitting out just to make the headlines or to impress unsuspecting members, is much worse and must be avoided if the labour movement is to amount to anything in this country."

Der Versuch sich nach zwei Seiten hin anzupassen, wird dadurch diktiert, daß die Erwartungen der Gewerkschaftsmitglieder an ihre Führer hoch sind, diese auch in der Regel über eine starke Stellung verfügen, die Regierung ihnen aber einige grundsätzliche Grenzen gezogen hat, die der breiten Masse der Gewerkschaftsmitglieder nicht deutlich sind. Im Falle der jeweiligen Úberschreitung dieser Grenzen wird der Repressionsapparat der Regierung eingesetzt ${ }^{31}$.

Schließlich wird auch gerade von Führern der NUPW immer wieder darauf verwiesen, daß die Gewerkschaften im Lande noch schwach, vor allem aber das Bewußtsein der breiten Mitgliedschaft noch viel zu wenig entwickelt sei, um für einen umfassenden Kampf mobilisiert zu werden ${ }^{32}$. Ganz sicher jedoch erschwert die rassische Strukturierung des politischen Systems die Politik der Gewerkschaften. Daß sie hier keine Änderung herbeizuführen suchten bzw. auch nicht wollten, ist wohl das Haupthindernis eines politischen Mobilisierungseffektes.

\section{Mobilisierungseffekte der Gewerkschaften?}

Auch wenn hier kein eingehendes Bild der Aktivitäten der malaysischen Gewerkschaften gezeichnet werden konnte, so ist doch der Nachweis geführt worden, daß die Gewerkschaften in Malaysia eine aktive Rolle spielen und daß sich ihre Aktivität in sichtbaren und zählbaren Ergebnissen niederschlägt. Okonomische und soziale Verbesserungen für Mitglieder, die nicht zuletzt auch auf andere gesellschaftliche Bereiche ausgestrahlit haben dürften, sind erreicht worden. Ihre Existenz regt sozusagen als Beispiel Arbeitnehmer an, in der Bildung von Gewerkschaften ein Mittel zu finden, das zur Uberwindung von Schwierigkeiten geeignet ist.

28 Dieses Argument wurde dem Verfasser von einem Gewerkschaftsführer der NUPW genannt, der im Wahlkampf 1974 den amtierenden Arbeitsminister gegen einen gewerkschaftlichen Gegenkandidaten unterstützte.

29 So ein Kabinettsminister gegenüber dem Verfasser.

30 New Straits Times, 18. 12. 1977.

31 Das ist vor allem der ,, Internal Security Act“, der z. B. 1979 ,,benützt“ wurde, um den Asien-Repräsentanten der Internationalen Transportarbeiterföderation wegen seiner Aufrufe zu einer internationalen Solidaritätsaktion mit streikenden Malaysiern mundtot zu machen. Vgl. New Straits Times, 18. 4. 1979, die dessen „Geständnis“ abdruckt.

32 Äußerungen gegenüber dem Verfasser. 
Hinsichtlich einer breiten politischen Mobilisierung der Arbeitnehmer kann auch nicht unbedingt von einem Mißerfolg gesprochen werden. Zwar haben es die von der Regierung bestimmten allgemeinen Bedingungen den Gewerkschaften unmöglich gemacht, selbst auf dem Felde der Arbeitsgesetzgebung erheblichen Einfluß auszuüben, es ist ihnen aber gelungen, der Regierung einzelne Zugeständnisse abzuringen ${ }^{33}$.

Auch ist es ihnen gelungen, durch ihre Kleinarbeit und ihre einzelnen Erfolge eine breite grundsätzliche Zustimmung zum Gewerkschaftskonzept im Lande durchzusetzen und bei den Arbeitnehmern das entsprechende Bewußtsein zu schaffen. Wohl der sichtbarste Beweis dafür ist, daß sie die Einführung politisch motivierter anti-gewerkschaftlicher Organisationen verhindern konnten.

Als nach der Notstandskrise 1969-1971 die politischen Parteien ,,Labour Bureaus“ einrichteten, schrieb der Generalsekretär des MTUC ${ }^{34}$ :

"Observing that the representatives of these parties have taken no pro-labour stand in the parliament, the General Council can hardly be blamed for thinking that their purpose for heralding themselves within their party organizations as groups who are friends and supporters of workers is nearly intended to mislead workers and to create confusion and disunity among them. This threat to workers' solidarity, coming as it is in a very subtle form, is by no means over and finished with and must not be underestimated."

Diese ,LLabour Bureaus“ sollten die Gewerkschaften ersetzen, schalteten sich auch auf Betriebsebene in Verhandlungen ein, konnten jedoch ein Wiedererstarken der Gewerkschaften nicht verhindern und blieben als Konkurrenzorganisationen erfolglos. Schon daran läßt sich ablesen, daß die malaysischen Gewerkschaften bis zu einem erheblichen Grad die Arbeitnehmer des Landes mobilisiert haben.

Andererseits hat die innere Struktur der Gewerkschaften sich vorerst noch so entwickelt, daß Arbeitnehmer unterer Einkommensgruppen unterrepräsentiert sind. Die Úberrepräsentanz bestimmter hochqualifizierter Führungsgruppen und deren Stabilität deuten an, daß die Mobilisierung der Gewerkschaften noch tiefer gehen und internsiver werden kann. Daß schließlich eine lebhafte Opposition innerhalb und außerhalb der Reihen des MTUC eine weitergehende Mobilisierung der gewerkschaftlichen Mitgliedschaft wünscht, zeigt an, daß es vorwärts drängende Kräfte gibt und daß die Gewerkschaften ,,leben“. Wenn diese Kräfte sich bisher nicht durchsetzen konnten, so zeigt dieses an, daß die vorsichtige Politik der Führung noch unterstützt wird - und sei es nur in Kenntnis des regierungseigenen Repressionsapparates.

Unter den obwaltenden malaysischen Umständen bestädigt sich da eigentlich die Erfahrung wohl aller Gewerkschaften - die seit der Massenstreikdebatte auch in Deutschland Maxime aller Gewerkschaftspolitik zu sein scheint-, daß nämlich es einen einfachen Weg zum Erfolg nicht gibt, Gewerkschaften zunächst danach streben, ihre Organisation zu sichern und zu erhalten und erst dann gegen die vielfältigen Widerstände kämpfen und diese oft in kleineren und mal in größeren Sprüngen überwinden.

\footnotetext{
33 So gelang es dem MTUC, der Regierung 1970/71 jene Notstandsverordnung wieder auszureden, die es den Gewerkschaften des öffentlichen Dienstes und der halbstaatlichen Einrichtungen untersagt hätte, Mitglieder des MTUC zu sein. 1973 ergänzte die Regierung nach langem Drängen des MTUC die Gesetzgebung dahingehend, daß Gewerkschaften von nun an in Wirtschaftsunternehmen investieren durften (was seit 1971 verboten war). 1976 wurde die „Employment Ordinance 1955“ reformiert, woebei eine Reihe von MTUC-Vorschlägen berücksichtigt wurden.

34 Malaysian Trades Union Congress, General Council Report 1971-1972, Petaling Jaya, S. 7.
} 


\section{Internal Terms of Trade Between Industry and Agriculture and Industrial Stagnation in India}

\section{By HANS-BERND SCHÄFER}

The article deals with the causes of the declining of the industrial growth rate in India. After the granting of independence in 1947, India followed a development strategy of rapid industrialization, with the bulk of investment going into heavy industry. This policy was - in terms of growth rates - very successful in the fifties and early sixties. However in the mid sixties, the growth rate declined sharply, and since then has never recovered.

The main thesis of this article is that the most important reason for this industrial depression was a rapid increase in the agricultural - versus industrial - prices. In the mid sixties, these prices rose by almost 50 per cent. In a country in which a wage earner spends most of his near subsistance income on agricultural products, this led to wage increases. These wage increases further delayed the possibilities for industrial profits and investment. The price rise for agricultural products indicated that neither by deliveries of the Indian farmers nor by commercial or concessional import could the growing non-agricultural labour force be maintained. Indian industry became locked in an internal terms-of-trade trap. On the other hand, high prices contributed to the remarkable performance of the Indian agricultural sector after 1967, making India self-sufficient in food. However high agricultural prices also raised the agricultural profit rate above the industrial profit rate. The consequence was a net transfer of savings into the agricultural sector. This is contradictory. to standard development economies, and again decreased the funds for industrial development.

\section{Trade Unions in Malaysia - Their Potential of Mobilization}

\section{By RUDIGER SiELAFF}

Trade Unions are social mass organizations designed to extend participation and change to people neglected by the socio-economic system. The model was developed in Europe. Social conditions, the political system and legislation in developing countries differ very much from nineteenth or early twentieth century Europe.

The author traces the development, the organizational structure and some of the achievements of Malaysian trade unions in order to describe the role, the achievements and the potential of Malaysian trade unions. The racial structure of the country which is particularly reflected in the political system and the different levels of development of different races, the colonial past and the conservative political leadership are some of the important factors influencing the development of trade unions in Malaysia.

Despite the legal restictions which limit the trade unions to the urban areas and to the plantations trade unions have organized roughly $20 \%$ of the employed people (neglecting the fact that the membership is always fluctuating strongly). The organization rate is much higher in public utilities, transport and services and growing strongly in the fairly new manufacturing industries. The organization rate among middle-class employees (technical and professional) is traditionally high, but it is growing very fast among urban industrial workers. The leadership of the unions is dominated by professionals and by professional trade unionists.

Racially the Indians are still dominating (largely due to the fact that they dominate the by far largest unions of the country, the National Union of Plantation Workers) with the influence of the Malay population growing. The Chinese who dominated the pre World War II trade union development never recovered their influence in trade unions after the Chinese domi- 
nated comunist influence in the trade unions was crushed in 1945 to 1948 . Besides the traditional trade union functions Malaysian trade unions have tried to strengthen their economic basis by forming economic ventures and cooperatives. They have not succeeded in organizing the rural areas.

The Malaysian trade unions have been able to influence some political decisions and to defend against the worst attempts to weaken them though their influence always has been limited. But they have been successful to uphold the multi-racial trade union ideology against the racially dominated political party structure. They have also been able to improve wages and working conditions with the most outstanding visible successes in the plantations.

\section{The Pursuit of Rights and Justice in International Law by the Developing Nations} By Emmanuel G. Bello

As far back as 1912, Oppenheim predicted with reasonable certitude that immeasurable progress was guaranteed to International Law because of the eternal moral and economic factors working in its favour. Allthough that prophesy was made nearly seventy years ago, yet it still appears difficult for certain individuals to accept that the enormous changes which have transformed world society have also had complex impact on International Law. There is a growing recognition that a new and uncertain epoch has arrived, and that the old system has definitely ended. A march is on to justify this change in order to accomplish within a short space of time what took the industrialised nations almost two centuries to achieve. The purpose of this study is to make a survey and analyse that presumption. 